\title{
Complications of revision spinal surgery
}

\author{
Kurt M. EichHolz, M.D., And Timothy C. Ryken, M.D. \\ Department of Neurosurgery, University of Iowa Hospitals and Clinics, Iowa City, Iowa
}

\begin{abstract}
Surgical treatment for symptomatic cervical and lumbar spondylosis has become prevalent in recent years. With this increased intervention, increasing numbers of patients experience persistent symptoms and require revision spinal surgery. Although many aspects of the workup and operation are similar for both primary and revision surgery, there are special considerations that must be examined when determining if a patient is a candidate for revision surgery.

Preoperative workup should include evaluation for spinal instability. Intraoperatively, scar tissue may complicate the procedure, and care must be taken to avoid incidental durotomy. The prognosis for a revision surgery can be predicted best by the patient's outcome after the primary surgery. As with any surgical procedure, patient selection is imperative for successful outcome.
\end{abstract}

KeY WORDS - revision surgery • complication • spine surgery

Surgical treatment for symptomatic spondylosis, involving either stenosis or radiculopathy, has become prevalent throughout the US. Many patients who have undergone spine surgery, however, frequently continue to experience symptoms that require revision surgery. In the lumbar spine, the failure rate has been reported to be as high as $37 \%$ after lumbar fusion and as high as $30 \%$ after lumbar surgery without fusion. ${ }^{8}$ Careful patient selection and meticulous surgical technique may decrease the need for revision surgery. Even with these precautions, however, the need for revision spinal surgery cannot be avoided in many cases.

When undertaking a revision spinal surgery, certain factors must be considered that are not present during a primary operation. General wound care is imperative because vascular supply may be compromised in revision surgeries. Epidural fibrosis and scarring can make a revision surgery significantly more difficult and can increase the risk of durotomy. Spinal fusion can lead to accelerated degeneration at adjacent levels, whereas extensive laminectomy can result in future instability.

In this article we discuss some of the general considerations in revision spinal surgery.

\section{REVISION SPINAL SURGERY}

\section{Preoperative Assessment}

Many patients who have undergone a primary spinal operation experience continued back or neck pain. Poste-

Abbreviation used in this paper: $\mathrm{MR}=$ magnetic resonance. rior dissection can cause significant myofascial pain due to the stripping of the paravertebral musculature from the posterior elements. Therefore, it is imperative to palpate the entire lumbar or cervical region in search of trigger points that may be amenable to injection. Careful palpation of the sciatic notch is also necessary to determine if sciatic notch syndrome is a possible cause of the patient's symptoms.

As always, a complete preoperative neurological examination is essential. In patients who have undergone previous lumbar or cervical discectomy, one must determine if the symptoms are at the same level and side or if the radiculopathy is related to a new lesion. ${ }^{11}$ It is also important to determine whether the patient improved after the primary operation. Outcome after the initial surgery can be a key indicator of prognosis after the revision procedure..$^{13}$ Stewart and Sachs ${ }^{13}$ have asserted that factors predisposing to failure of revision surgery include patient age, the number of levels involved in the primary surgery, and pain-free interval after primary surgery.

\section{Radiographic Studies}

In addition to the physical examination, radiographic/ neuroimaging studies must be obtained and critically examined. In patients who have undergone a previous spinal surgery, plain x-ray films must be obtained in flexion and extension to evaluate for instability. In cases in which translation is demonstrated in flexion or extension when this finding was not present prior to the initial surgery, the patient may be a candidate for fusion. Indeed, the authors of several studies have advocated lumbar fusion in the treatment of recurrent disc herniations associated with 
concurrent vertebral instability. ${ }^{7,9,15}$ Flexion and extension radiographs should also be obtained prior to any primary spinal surgery. Although instability does not usually result from a standard cervical or lumbar laminectomy, it is more likely to develop in patients in whom ligamentous instability is demonstrated prior to the primary surgery. Fritsch, et al., ${ }^{7}$ reported that instability was documented in up to $60 \%$ of patients who have undergone multiple revisions.

Magnetic resonance imaging is also a vital part of the preoperative workup. In the potential candidate for revision surgery, the MR imaging should include $T_{2}$ - and $T_{1}$ weighted sequences, with and without administration of Gd. Gadolinium administration can help the clinician differentiate between recurrent disc herniations and scar tissue. ${ }^{3}$ Herniated disc fragments should not enhance on Gdbased MR images, whereas scar tissue will enhance. This is important because revision surgery for scar tissue-related symptoms most often has poor results.

Postmyelography computerized tomography scanning can also be very useful in determining a patient's need for revision spinal surgery. Although this modality is not used as often as it was prior to the advent of MR imaging, it can provide useful information not readily apparent on other radiographic modalities. Postmyelography computerized tomography scanning can demonstrate the anatomy of the nerve root sleeves as they exit the neural foramina. It can also reveal nerve root clumping or disturbances of flow of the contrast material that can indicate arachnoiditis.

\section{Surgery-Related Issues}

In patients who have undergone several prior spinal surgeries, devascularization of the tissue is frequently involved with the surgical wound. This devascularization can impede wound postoperative healing and may predispose to wound infection. Perioperative antibiotic therapy should be conducted. Meticulous care must be taken during wound closure to ensure that appropriate fascial layers are reapproximated, and closure should be undertaken in several layers by using resorbable (vicryl) sutures. Skin sutures should be left in place for a longer period of time (10-14 days) than for a primary operation.

When performing a primary cervical or lumbar laminectomy, care should be taken not to destabilize the facet joints. This is especially important in patients in whom ligamentous instability is demonstrated; in this situation, the surgeon should consider performing arthrodesis. Prior hemilaminotomy, either in the cervical or lumbar region, should not predispose to instability. ${ }^{7}$

\section{Adjacent-Level Degeneration}

With the advent of techniques for anterior cervical surgery ${ }^{4,12}$ and the recent popularity of anterior arthrodesis, complications have been noted to develop in the longterm follow-up period. Anterior decompression not involving fusion has been found to predispose to kyphotic deformity or pseudarthrosis. ${ }^{5,6}$ This may lead to central canal stenosis and resultant myelopathy. Anterior arthrodesis has also been shown to predispose to adjacent-level degeneration. The immobilization of one motion segment may cause increased stress at the adjacent level, most fre- quently at the level superior to the fusion (Fig. 1). Spondylosis or disc herniation at the level above a previous fusion may cause new radiculopathy or myelopathy. In this situation, decompression and extension of the previous fusion may be required. ${ }^{5}$

Extensive cervical laminectomy may result in kyphotic deformity (Fig. 2). Postlaminectomy kyphosis may cause axial neck pain and myelopathic symptoms. In this setting, multilevel combined anterior-posterior reconstruction is necessary to reestablish normal cervical alignment.

\section{Scar Tissue and Incidental Durotomy}

Epidural fibrosis and scarring significantly increase the difficulty of revision spinal surgery, especially in the lumbar region. Extensive epidural scar tissue and dural adhesions increase the likelihood of incidental durotomy. These may not be recognized at the time of surgery. The surgeon must establish a plane between the scar tissue and the dura mater. Implantable barriers have been devised to prevent the formation of scar tissue ${ }^{1}$ however, they have not been proven effective and are not widely used in the US.

Incidental durotomy can create many problems during spinal surgery. The authors of several studies have shown that the incidence of durotomy in revision spinal surgery is higher than in primary surgery. ${ }^{2,10,14}$ Cammisa, et al., ${ }^{2}$ reported an overall $3.1 \%$ incidence of durotomy in 2144 spinal procedures; however, its incidence in primary surgeries was 1 to $3.1 \%$, whereas the incidence in revision surgery was $8.1 \%$. Unfortunately, no statistical analysis was performed in their study. Patients in whom dural tears were recognized and treated appropriately at the time of surgery did not suffer any significant long-term sequelae. Small incidental durotomies that are not noted at the time

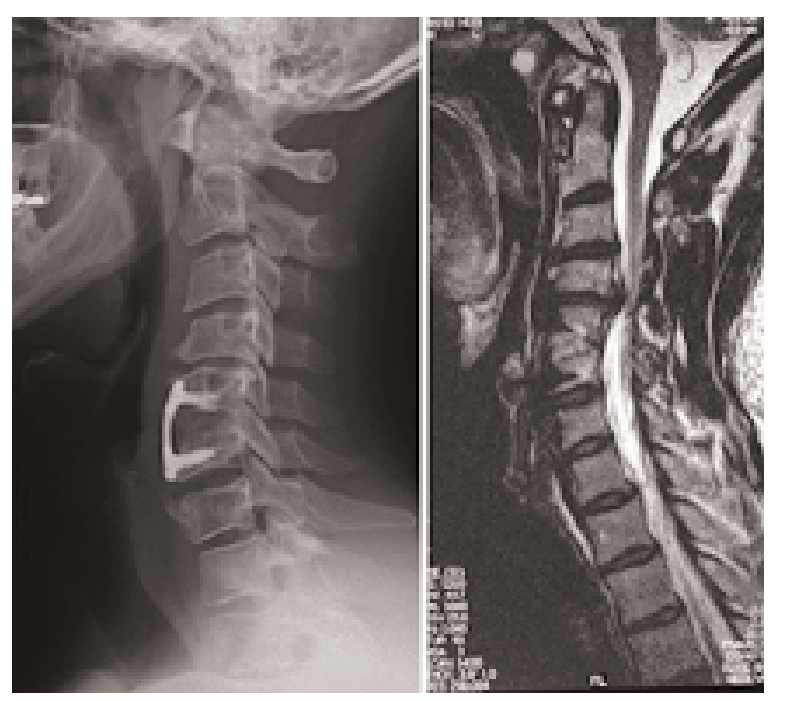

Fig. 1. Imaging studies obtained in a 37-year-old man who had undergone C5-6 anterior decompression and arthrodesis. He presented 4 years later with neck pain and cervical myelopathy. Left: Lateral cervical radiograph. Right: Parasagittal $\mathrm{T}_{2}$-weighted MR image. Note the large disc herniation at $\mathrm{C} 4-5$ directly above the previous arthrodesis. This patient required $\mathrm{C} 4-5$ anterior decompression and extension of the fusion. 

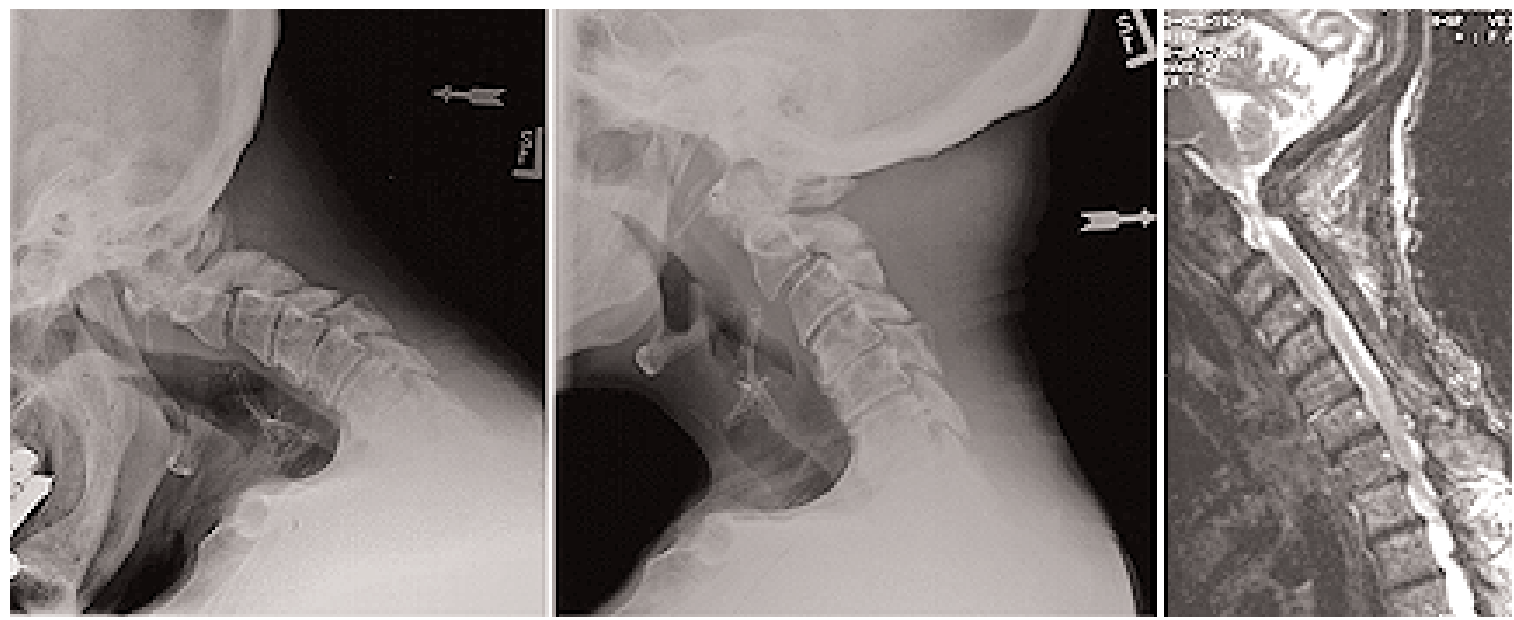

Fig. 2. Imaging studies obtained in a patient with kyphosis after undergoing an extensive cervical laminectomy. Left: Lateral cervical radiograph in flexion. Center: Lateral cervical radiograph in extension. Right: Sagittal $\mathrm{T}_{2}-$ weighted MR image.

of surgery can lead to formation of pseudomeningoceles. ${ }^{2}$ If a small incidental durotomy is not treated appropriately at the time of surgery, a pseudomeningocele may develop (Fig. 3). If the pseudomeningocele is small and asymptomatic, it may be treated with conservative therapy. Bed rest and possibly lumbar drainage may be of benefit. If, however, the pseudomeningocele is large, causing back

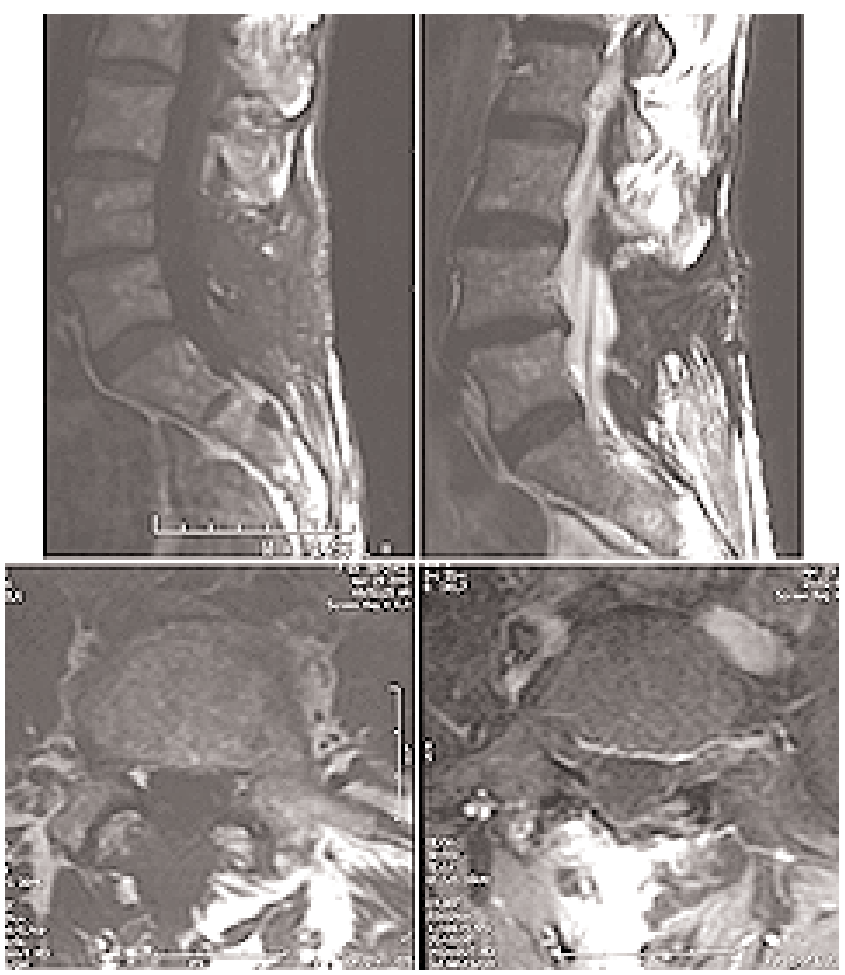

Fig. 3. Upper Left and Right: Sagittal and axial $\mathrm{T}_{1}$ - (upper left and lower right) and $\mathrm{T}_{2}$-weighted (upper right and lower left) $\mathrm{MR}$ images demonstrating a postoperative pseudomeningocele. pain, or if there is the presence of drainage from the wound, reexploration must be performed.

At the time of surgery, the wound may be irrigated and a valsalva maneuver performed to evaluate for the presence of small incidental durotomies. Closure of small dural defects may be conducted using No. 5-0 or 6-0 prolene suture. If a durotomy is made on the lateral aspect of the thecal sac, gaining access for primary repair may be difficult. In this case, fibrin glue or other dural substitutes may be used to assist with dural closure. The patient should be undergo bed rest while lying flat for at least 24 hours to facilitate closure and to prevent formation of pseudomeningocele. The wound should be carefully inspected in the postoperative period for signs of compromise. The clinician should have a low threshold for reimaging or reexploration in the presence of clear drainage from the surgical site. ${ }^{2}$

\section{CONCLUSIONS}

There are many factors involved in revision spinal surgery that the clinician must consider. As with any surgical procedure, proper patient selection is imperative in achieving a successful outcome. In addition, the preoperative workup in the potential candidate for revision spinal surgery should include evaluation for scar tissue, instability, and possible new lesions. Myofascial pain should also be considered, and only candidates in whom adequate course of conservative therapy has failed should be considered for revision surgery.

The outcome of revision surgery may be predicted by the patient's outcome after the primary surgery. These factors should be taken into account when considering revision spinal surgery.

\section{References}

1. Brotchi J, Pirotte B, De Witte O, et al: Prevention of epidural fibrosis in a prospective series of 100 primary lumbo-sacral dis- 
cectomy patients: follow-up and assessment at re-operation. Neurol Res 21 (Suppl 1):S47-S50, 1999

2. Cammisa FP Jr, Girardi FP, Sangani PK, et al: Incidental durotomy in spine surgery. Spine 25:2663-2667, 2000

3. Cinotti G, Roysam GS, Eisenstein SM, et al: Ipsilateral recurrent lumbar disc herniation. A prospective, controlled study. J Bone Joint Surg Br 80:825-832, 1998

4. Cloward RB: The anterior approach for removal of ruptured cervical disks. J Neurosurg 15:602-617, 1958

5. Coric D, Branch CL Jr, Jenkins JD: Revision of anterior cervical pseudarthrosis with anterior allograft fusion and plating. J Neurosurg 86:969-974, 1997

6. Etminan M, Girardi FP, Khan SN, et al: Revision strategies for lumbar pseudarthrosis. Orthop Clin North Am 33:381-92, 2002

7. Fritsch EW, Heisel J, Rupp S: The failed back surgery syndrome: reasons, intraoperative findings, and long-term results: a report of 182 operative treatments. Spine 21:626-633, 1996

8. Frymoyer JW, Matteri RE, Hanley EN, et al: Failed lumbar disc surgery requiring second operation. A long-term follow-up study. Spine 3:7-11, 1978

9. Glassman SD, Minkow RE, Dimar JR, et al: Effect of prior lumbar discectomy on outcome of lumbar fusion: a prospective analysis using the SF-36 measure. J Spinal Disord 11: 383-388, 1998

10. Jones AA, Stambough JL, Balderston RA, et al: Long-term results of lumbar spine surgery complicated by unintended incidental durotomy. Spine 14:443-446, 1989

11. Ozgen S, Naderi S, Ozek MM, et al: Findings and outcome of revision lumbar disc surgery. J Spinal Disord 12:287-292, 1999

12. Smith GW, Robinson RA: The treatment of certain cervicalspine disorders by anterior removal of the intervertebral disc and interbody fusion. J Bone Joint Surg Am 40:607-624, 1958

13. Stewart G, Sachs BL: Patient outcomes after reoperation on the lumbar spine. J Bone Joint Surg Am 78:706-711, 1996

14. Wang JC, Bohlman HH, Riew KD: Dural tears secondary to operations on the lumbar spine. Management and results after a two-year-minimum follow-up of eighty-eight patients. J Bone Joint Surg Am 80:1728-1732, 1998

15. Wong CB, Chen WJ, Chen LH, et al: Clinical outcomes of revision lumbar spinal surgery: 124 patients with a minimum of two years of follow-up. Chang Gung Med J 25:175-182, 2002

Manuscript received July 14, 2003.

Accepted in final form August 12, 2003.

Address reprint requests to: Timothy C. Ryken, M.D., Department of Neurosurgery, University of Iowa Hospitals and Clinics, 200 Hawkins Drive, Iowa City, Iowa 52242. timothy-ryken@ uiowa.edu.. 